\title{
A Simple Method for Normalization of Aortic Contractility
}

\author{
Lexiao Jin ${ }^{a, b}$ Alexandra Lipinski ${ }^{c, d}$ Daniel J. Conklin ${ }^{b-d}$ \\ ${ }^{a}$ Department of Anesthesiology, Critical Care and Pain Medicine, The Second Affiliated Hospital and Yuying \\ Children's Hospital of Wenzhou Medical University, Wenzhou, China; ${ }^{b}$ Department of Pharmacology and \\ Toxicology, University of Louisville, Louisville, KY, USA; ' Institute of Molecular Cardiology, Department of \\ Medicine, University of Louisville, Louisville, KY, USA; 'Diabetes and Obesity Center, Department of Medicine, \\ University of Louisville, Louisville, KY, USA
}

\section{Keywords}

Aorta · Blood vessel · Isometric contraction · Myography ·

Phenotypic switch · Vascular smooth muscle

\begin{abstract}
Vascular contractile function changes in proliferative vascular diseases, e.g. atherosclerosis, and is documented using isolated blood vessels; yet, many laboratories differ in their approach to quantification. Some use raw values (e.g., mg, $\mathrm{mN}$ ); others use a "percentage of control agonist" approach; and others normalize by blood vessel characteristic, e.g. length, mass, etc. A lack of uniformity limits direct comparison of contractility outcomes. To address this limitation, we developed a simple 2-step normalization method: (1) measure blood vessel segment length $(\mathrm{mm})$, area $\left(\mathrm{mm}^{2}\right)$ and calculate volume $\left(\mathrm{mm}^{3}\right)$; then, (2) normalize isometric contraction $(\mathrm{mN})$ by segment length and volume. Normalized aortic contractions but not raw values were statistically different between normal chow and high-fat diet-fed mice, supporting the practical utility and general applicability of normalization. It is recommended that aortic contractions be normal-
\end{abstract}

(c) 2018 S. Karger AG, Basel

E-Mail karger@karger.com

www.karger.com/jvr ized to segment length and/or volume to reduce variability, enhance efficiency, and to foster universal comparisons across isometric myography platforms, laboratories, and experimental settings.

(c) 2018 S. Karger AG, Basel

\section{Introduction}

Cardiovascular disease is the leading cause of death worldwide. Atherosclerosis is the primary form of cardiovascular disease that increases the risk of myocardial infarction, stroke, and peripheral arterial disease. An underlying mechanism of atherosclerosis involves a "phenotypic switch" in vascular smooth muscle cells (VSMC) that transcriptionally moves VSMC from a contractile to a proliferative or "synthetic phenotype" $[1,2]$. Thus, measuring contractility in isolated blood vessels provides functional evidence of phenotypic change reflective of the

L.J. anf A.L. are co-first authors of this study.
Prof. Daniel J. Conklin

Diabetes and Obesity Center, Institute of Molecular Cardiology 580 S. Preston Street, Delia Baxter, Rm. 404E

University of Louisville, Louisville, KY 40202 (USA)

E-Mail dj.conklin@louisville.edu 
underlying local cellular and biochemical alterations such as in atherosclerosis. Thus, laboratories around the world measure vascular contractile function in vitro, wherein an isolated blood vessel, e.g. aorta, is contracted with an agonist, e.g. phenylephrine (PE), endothelin-1, U46,619 - a thromboxane $\mathrm{A}_{2}$ analog, etc... and data reported [3-5]. The widespread use of in vitro vascular contractility assays is revealed in PubMed search using the terms "vascular" and "dysfunction," which netted 214,358 hits, indicating a sizeable research investment.

Although raw isometric data reflect vessel contractility, raw values (e.g., $\mathrm{mg}$ or $\mathrm{mN}$ tension) [6] cannot be compared across different laboratories due to obvious differences in the size of the blood vessel segment (e.g., length, diameter), species, animal size, age, diet, and disease state as well as the specific blood vessel or blood vessel location (e.g., thoracic vs. abdominal aorta). Additionally, different platforms or myography systems (e.g., DMT) are used to assess isometric force production. So, even if two laboratories use the same system, the reporting of data can make it difficult to compare contractility due to dissimilar units ( $\mathrm{mg}$ vs. $\mathrm{mN}$ ) or a different data normalization procedure, e.g. percent of control agonist such as percent contraction of high-potassium solution) $[7,8]$ or differential normalization, e.g. $\mathrm{mg}$ tension/cross-sectional area; $\mathrm{mg}$ tension/ $\mathrm{mg}$ wet weight; $\mathrm{mN} / \mathrm{mm}$ length $[3,4,9]$. To address this obvious limitation and to foster more across-study and laboratory comparisons, aortic contractions were normalized by several easily measured parameters.

Several criteria need to be met to establish a universal normalization method. For example, the method needs to be accessible, and thus, it should not require costly equipment that may exclude users. It should be simple enough that all users can implement the method. Moreover, the method should be cost effective in that the time spent performing should be worth the effort. Because current laboratories already use some form of normalization (e.g., as "\% of control agonist") [8], a new approach must be cost effective and enhance data quality over an existing practice. Finally, the method should foster data comparisons for both cross-laboratory fertilization and greater biological understanding. To this end, we offer a simple and cost-effective method for normalization of vascular contractility that requires minimal equipment and effort on the user's behalf, yet refines the measurement of aortic contraction to a degree wherein even modest changes in contractility (e.g., due to a treatment, aging, diet, etc...) are more readily discerned. Contractility data that are universally normalized can be shared and compared between laboratories around the globe. Adoption of this ap- proach may improve assessment of the biological actions of drugs, diets or toxicants, for example, and thus, minimize overall use of resources.

\section{Materials and Methods}

\section{Rodents}

Male C57BL/6 mice were purchased from The Jackson Laboratory (Bar Harbor, ME, USA) or raised in house. Mice were fed normal chow (NC; 13\% kcal fat, Rodent Diet 5010; LabDiet, St. Louis, MO, USA) or high-fat (HFD; 42\% kcal fat, TD.88137; Harlan Teklad, Madison, WI, USA) diet from 8 to 20 weeks of age. Male Sprague-Dawley rats (wild type [WT] and low-density lipoprotein receptor deficient [LDLR-KO]) were obtained from SAGE (Sigma-Aldrich) and fed NC diet up to 1 year of age [10]. Rodents were treated according to APS's Guiding Principles in the Care and Use of Animals, and all protocols were approved by University of Louisville IACUC. All rodents were anesthetized with sodium pentobarbital (i.p., $150 \mathrm{mg} / \mathrm{kg}$ ) and exsanguinated via right ventricle blood draw.

\section{Equipment}

The following equipment was used: digital handheld microscope (zORB, Carson, New York, NY, USA); scientific ruler (1 mm increments; Fine Science Tools); 10\% neutral buffered formalin solution (NBF; Sigma); Falcon ${ }^{\circledR} 12$-well, clear, flat bottom plate (Corning Costar); Image (NIH free imaging software); razor blades; dental wax (Z Pink); general surgical tools (scissors, forceps); microfuge tubes; organ bath myography system; and, microbalance. All chemicals were purchased from Sigma-Aldrich unless stated otherwise.

\section{Aorta Isolation and Organ Bath Systems}

Aortas were isolated via ventral thoracotomy and placed into cold phosphate-buffered solution (PSS) containing (in $\mathrm{mM}$ ): $\mathrm{NaCl}$, 130; $\mathrm{KCl}, 4.7 ; \mathrm{MgSO}_{4} \cdot 7 \mathrm{H}_{2} \mathrm{O}, 1.17 ; \mathrm{KH}_{2} \mathrm{PO}_{4}, 1.18 ; \mathrm{NaHCO}_{3}, 14.9$; $\mathrm{CaCl}_{2}$, 2.0; glucose, 5.0; $\mathrm{pH}$ 7.4. High-potassium ( $\mathrm{HI} \mathrm{K}^{+}$) PSS contained the following (in $\mathrm{mM}$ ): $\mathrm{NaCl}, 34.7 ; \mathrm{KCl}, 100 ; \mathrm{MgSO}_{4} \cdot 7 \mathrm{H}_{2} \mathrm{O}$, 1.17; $\mathrm{KH}_{2} \mathrm{PO}_{4}, 1.18 ; \mathrm{NaHCO}_{3}, 14.9 ; \mathrm{CaCl}_{2}$, 2.0; glucose, 5.0; $\mathrm{pH}$ 7.4. Distal thoracic aorta (located $0.8-1 \mathrm{~cm}$ from aortic arch apex) segments were cut from each aorta $(2-4 \mathrm{~mm})$, cleaned of perivascular adipose tissue, and mounted in one of two systems: (1) horizontal pin (DMT, Ann Arbor, MI, USA); or, (2) vertical wire system. In the horizontal pin system, a short, aortic segment $(\approx 2 \mathrm{~mm})$ was mounted on stainless steel pins in a $5-\mathrm{mL}$ heated $\left(37^{\circ} \mathrm{C}\right)$ organ bath. One pin was connected to an isometric strain gauge transducer, and the other was attached to a micrometer. Transducer signals were fed directly into a PC with LabChart software (v. 7 or 8; ADI). In the vertical wire system, an aortic segment $(3-5 \mathrm{~mm})$ was placed on wire hooks (100- $\mu \mathrm{m}$ diameter tungsten wire) with a hook connected to an isometric strain gauge transducer (Kent Scientific, Litchfield, CT) and the other attached to a fixed support glass rod in a 15 - $\mathrm{mL}$ heated $\left(37^{\circ} \mathrm{C}\right)$ organ bath. Transducer signals were fed into an 8-channel PowerLab A/D converter and recorded on a PC using LabChart software (v. 4.3.2; ADI). Prior to each use, each system was calibrated using standardized weights. In both systems, after 10 min without tension, aortic rings were equilibrated to $\approx 9.81 \mathrm{mN}$ $(\approx 1 \mathrm{~g})$ loading tension over $30 \mathrm{~min}$ before agonist stimulation [3]. 
Fig. 1. Images of method for aortic parameter data acquisition. a Digital images of blood vessel segment length (mm) acquired with digital mini-microscope camera. b Digital image of blood vessel crosssection overlaid with digital outlines of outer and inner circumferences (NIH ImageJ). The outer area (OA) minus the inner area (IA; lumen) is the segment cross-sectional area $\left(\mathrm{mm}^{2}\right)$. c Calculation of blood vessel segment volume where, volume = length $(\mathrm{mm}) \times$ cross-section area $\left(\mathrm{mm}^{2}\right)$ in $\mathrm{mm}^{3}$.

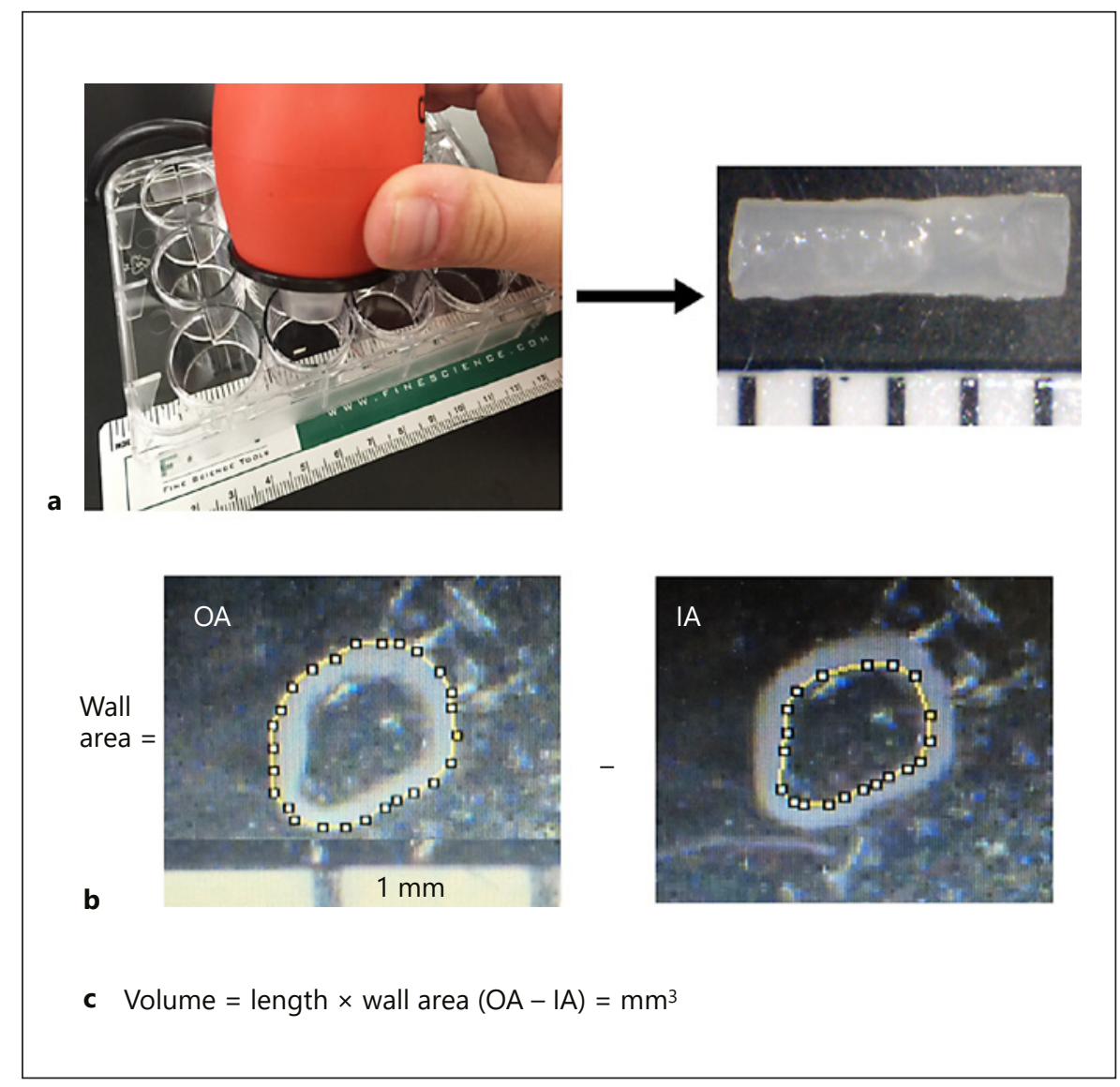

\section{Statistics}

Values are expressed as mean \pm SD or \pm SE (where appropriate). Comparisons between 2 groups (e.g., horizontal vs. vertical system; user 1 vs. user 2 ; etc...) were done with a paired or unpaired $t$ test as appropriate. $p<0.05$ was considered significant.

\section{Results and Discussion}

\section{Acquisition of Aortic Segment Parameters}

Normalization of contractions was done using a simple, sequential, multistep process that is described pictorially in Figure 1, and described here as a general protocol: (1) immediately formalin-fix a blood vessel segment; (2) obtain wet weight (to $0.1 \mathrm{mg}$; dab extra fluid on tissue paper); (3) image segment lengthwise with scientific ruler (Fig. 1a); (4) cut (with razor blade) and image (with handheld digital microscope) a thin $(0.5 \mathrm{~mm})$ blood vessel cross-section (x-sec) with a ruler in the image (Fig. 1b); (5) using ImageJ, quantify segment length $(\mathrm{mm})$ and $\mathrm{x}-$ sec area $\left(\mathrm{mm}^{2}\right)$ (Fig. 1c); and, (6) calculate segment vol- ume $\left(\mathrm{mm}^{3}\right)$ as the product of length $(\mathrm{mm})$ and $\mathrm{x}$-sec area $\left(\mathrm{mm}^{2}\right)$, and then normalize isometric contractions by segment length $(\mathrm{mN} / \mathrm{mm})$, $\mathrm{x}$-sec area $\left(\mathrm{mm}^{2}\right)$, and volume $\left(\mathrm{mN} / \mathrm{mm}^{3}\right)$.

\section{Aortic Function}

Aortic vascular contractile function was assessed using both a horizontal and a vertical isometric tension organ bath system as reported previously [3]. To illustrate the utility of the normalization protocol, the distal thoracic aorta of a mouse was sectioned into 2 obvious, unequal lengths. A shorter piece (1.5-2.5 mm) was mounted in the horizontal pin system and a longer piece $(3-4 \mathrm{~mm})$ was mounted in the vertical system. Both segments were equilibrated similarly $(\approx 9.81 \mathrm{mN}$ tension). As intended in this "proof of principle" example, the absolute (raw) tension of aortic contractions $(\mathrm{mN})$ induced either by cumulative concentrations of PE ( $1 \mathrm{nM}$ to $10 \mu \mathrm{M}$; Fig. 2a) or by high-potassium solution ( $100 \mathrm{mM}$; Fig. $2 \mathrm{~b}$ ) was significantly different between the two due to the intentional difference in blood vessel segment size $(\approx 2 \times)$. 


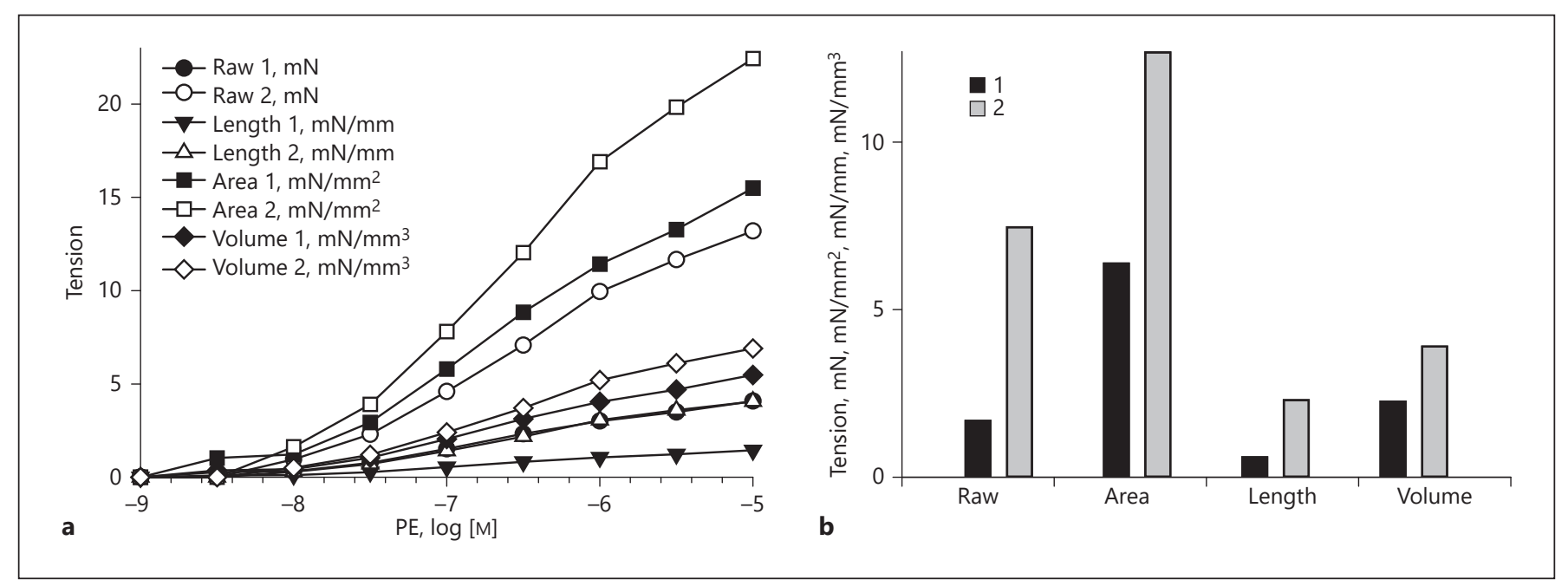

Fig. 2. Normalization of vascular contractility data. Contraction data $(\mathrm{raw}, \mathrm{mN})$ and data normalized by aorta cross-sectional area $\left(\mathrm{mN} / \mathrm{mm}^{2}\right)$, length $(\mathrm{mN} / \mathrm{mm})$, and volume $\left(\mathrm{mN} / \mathrm{mm}^{3}\right)$ were plotted. To generate tension data, two aortic segments were exposed to contractile agents: phenylephrine (PE; $1 \mathrm{nM}$ to $10 \mu \mathrm{M})(\mathbf{a})$ and high- potassium (100 mM) buffer (b); in a horizontal pin (DMT; 1, filled symbols), or a vertical wire (2, open symbols) organ bath system. After experimentation, length and cross-sectional area of formalinfixed blood vessel segments were measured and the segment volume calculated (volume $=$ length $\times$ cross-section area; see Fig. 1 ).
Table 1. Thoracic aorta segment parameters from C57BL/6 mice fed normal chow $(n=9)$ or high-fat diet $(n=10)$ for 12 weeks

\begin{tabular}{lcc}
\hline Parameter & Diet & \\
\cline { 2 - 3 } & normal chow & high-fat diet \\
\hline Wall area, $\mathrm{mm}^{2}$ & $0.246 \pm 0.024$ & $0.225 \pm 0.020$ \\
Length, $\mathrm{mm}$ & $3.96 \pm 0.56$ & $4.37 \pm 0.41$ \\
Volume, $\mathrm{mm}^{3}$ & $0.976 \pm 0.178$ & $0.990 \pm 0.171$ \\
\hline
\end{tabular}

Values $=$ means \pm SE; $n=9,10$ mice, respectively

\section{Normalization of Aortic Contraction}

After acquisition of aortic parameters, cumulative PE and high potassium-induced contractions were normalized to $\mathrm{x}$-sec area, length, and volume (Fig. 2). Normalization of raw tension by segment volume $\left(\mathrm{mN} / \mathrm{mm}^{3}\right)$ resulted in the most similar PE-induced tension curves (see curves of "volume 1" and "volume 2"; Fig. 2a). Whereas normalization of raw values to either $\mathrm{x}$-sec area or length was beneficial in reducing the difference between "raw 1" and "raw 2 " values, but neither was superior to normalization by volume. Notably, in a separate study, a group used a standardized mouse aorta segment length $(3 \mathrm{~mm})$ to normalize contractility and arrived at values similar to ours, thus, supporting the idea that normalized contractions even to one
Table 2. Normalization of high potassium-induced (100 mM) isometric contractions in thoracic aortas isolated from male mice fed either normal chow (NC) or high fat diet (HFD) for 12 weeks

\begin{tabular}{lllll}
\hline Group & $\mathrm{mN}$ & $\mathrm{mN} / \mathrm{mm}$ & $\mathrm{mN} / \mathrm{mm}^{2}$ & $\mathrm{mN} / \mathrm{mm}^{3}$ \\
\hline NC & $8.26 \pm 1.03$ & $2.08 \pm 0.22$ & $33.16 \pm 3.32$ & $8.37 \pm 0.71$ \\
HFD & $5.54 \pm 0.61$ & $1.26 \pm 0.13$ & $24.61 \pm 2.60$ & $5.66 \pm 0.57$ \\
\hline$p$ value & 0.0330 & 0.0047 & 0.0562 & 0.0079
\end{tabular}

Values $=$ mean $\pm \mathrm{SE} ; n=9,10$ mice, respectively; $p$ values shown are results of unpaired $t$ tests between NC and HFD groups.

dimension (e.g., length; $\mathrm{mN} / \mathrm{mm}$ ) likely are better than no normalization (Fig. 2a) [11]. Normalization efficacy was independent of the contractile agonist because a similar effect was observed when $\mathrm{HI} \mathrm{K}^{+}$-induced maximal tension was normalized to $\mathrm{x}$-sec area, length and volume (Fig. 2b). As for PE, normalization to volume appeared to perform better than using either length or area alone.

In a second example, real-world contractility was measured in aortas isolated from C57BL/6 mice fed either an NC ( $13 \% \mathrm{kcal}$ fat) or HFD ( $42 \% \mathrm{kcal}$ fat) for 12 weeks. As all aorta segments had similar lengths, $\mathrm{x}$-sec areas, and thus, volumes (Table 1), direct comparison of raw contractility values revealed that 12 weeks of HFD appeared 

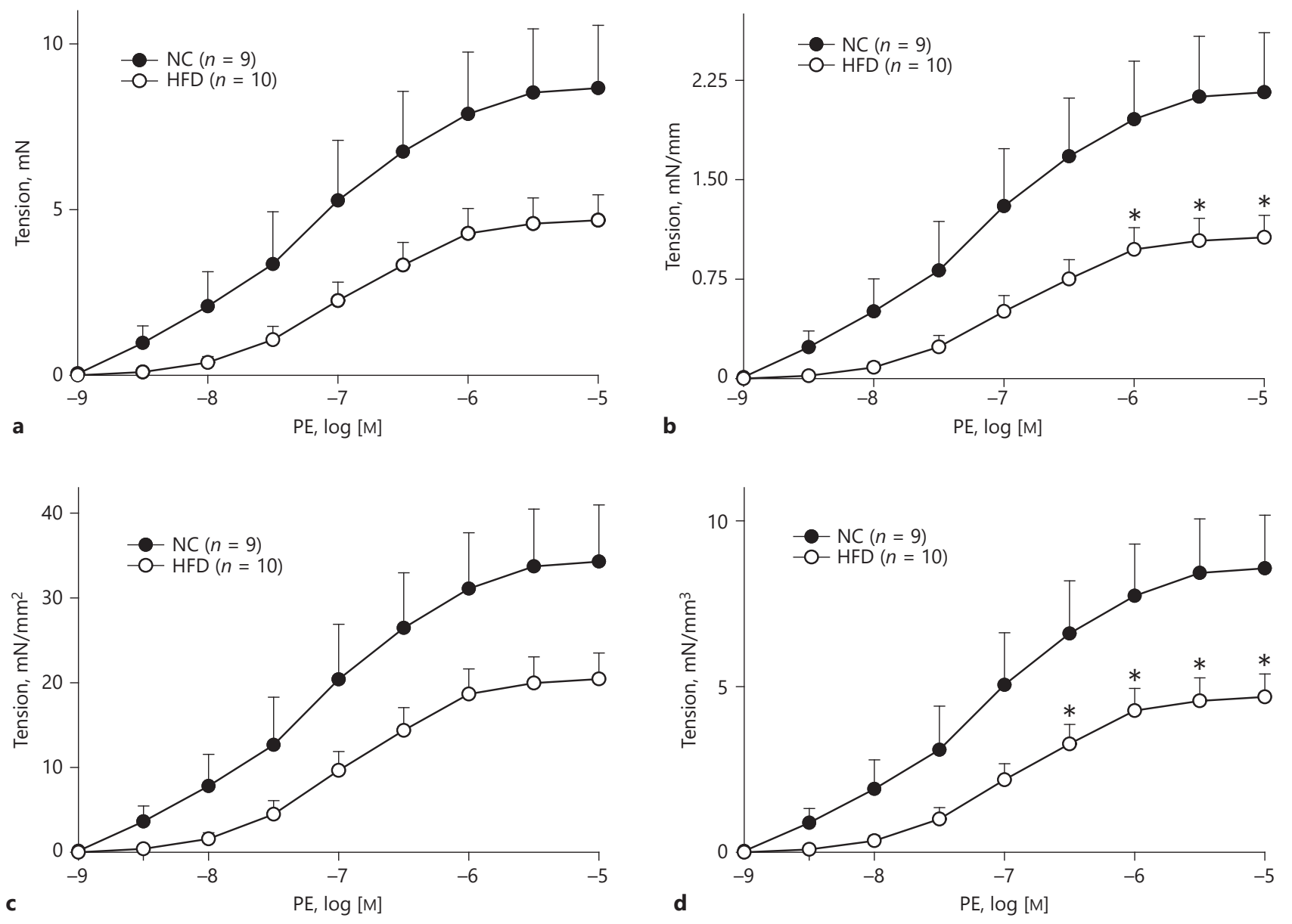

Fig. 3. Normalization of vascular contractility of aortas from C57BL/6 mice fed normal chow (NC; $n=9$ mice) or high-fat diet (HFD; $n=10$ mice) for 12 weeks. Phenylephrine (PE)-induced raw contraction data $(\mathbf{a} ; \mathrm{mN})$ and raw data normalized by aorta length $(\mathbf{b} ; \mathrm{mN} / \mathrm{mm}), \mathrm{x}$-sec area $\left(\mathbf{c} ; \mathrm{mN} / \mathrm{mm}^{2}\right)$, and volume $\left(\mathbf{d} ; \mathrm{mN} / \mathrm{mm}^{3}\right)$. Values $=$ mean \pm SE. $n=9$ or 10 aortas $(1$ aorta per mouse $) .{ }^{*} p<0.05$ between NC and HFD.

to decrease PE-induced contractions relative to aortas of NC-fed mice, yet there were no statistically significant differences (Fig. 3a). To assess whether normalization could unmask differences in contractility, raw values were normalized by length (Fig. 3b), area (Fig. 3c), and volume (Fig. 3d). When normalized to aortic segment length or volume, contractions were statistically different between NC and HFD mice (Fig. 3b, d). Overall, these data indicate that normalization of aortic contractility enhanced detection of statistically significant changes induced by HFD (Fig. 3b, d). Similarly, aortic contractions induced by $100 \mathrm{mM} \mathrm{HI} \mathrm{K}{ }^{+}$buffer were also significantly
Table 3. Thoracic aorta segment parameters from wild-type (WT) and LDLR-null (LDLR-KO) 1-year-old Sprague-Dawley rats

\begin{tabular}{lcc}
\hline Parameter & \multicolumn{2}{l}{ Strain } \\
\cline { 2 - 3 } & WT & LDLR-KO \\
\hline Wall area, mm $\mathrm{mm}^{2}$ & $1.251 \pm 0.213$ & $1.168 \pm 0.224$ \\
Length, $\mathrm{mm}$ & $3.07 \pm 0.49$ & $2.74 \pm 0.48$ \\
Volume, $\mathrm{mm}^{3}$ & $3.90 \pm 1.055$ & $3.269 \pm 1.138$ \\
\hline
\end{tabular}

Values $=$ means \pm SE. $n=5,6$ rats, respectively. 

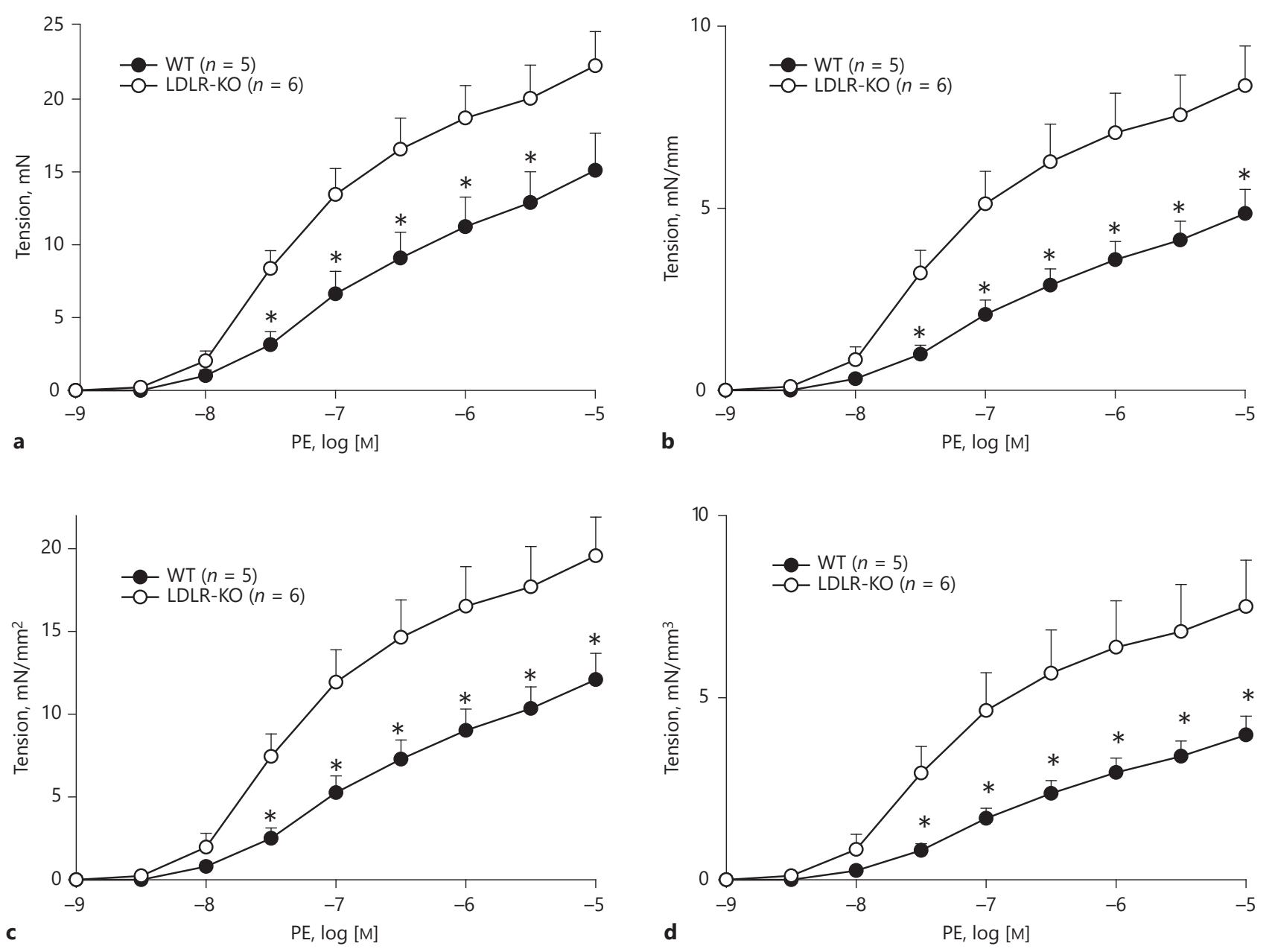

Fig. 4. Normalization of vascular contractility of aortas from Sprague-Dawley wild type (WT; $n=5$ rats) and LDLR-KO rats ( $n=6$ rats) fed normal chow for 1 year. Phenylephrine (PE)-induced raw contraction data $(\mathbf{a} ; \mathrm{mN})$ and raw data normalized by aorta length $(\mathbf{b} ; \mathrm{mN} / \mathrm{mm}), \mathrm{x}$-sec area $\left(\mathbf{c} ; \mathrm{mN} / \mathrm{mm}^{2}\right)$, and volume $\left(\mathbf{d} ; \mathrm{mN} / \mathrm{mm}^{3}\right)$. Values $=$ mean \pm SE. $n=5$ or 6 aortas ( 1 aorta per rat). ${ }^{*} p<0.05$ between WT and LDLR-KO rats.

between NC and HFD-fed groups both as raw values and when normalized by length and volume (Table 2).

Another real-world example was performed using aortas isolated from 1-year old Sprague-Dawley rats that were WT or LDLR-KO. As all aorta segments had similar lengths, $\mathrm{x}$-sec areas, and thus, volumes (Table 3), direct comparison of raw contractility values revealed that genetic deletion of LDLR increased PE-induced contractions relative to aortas of WT rats (Fig. 4a). To assess whether normalization could alter the statistical differences observed, raw values were normalized by length (Fig. 4b), area (Fig. 4c), and volume (Fig. 4d). Normaliza- tion of raw contractility data to aortic segment length or volume yet not area enhanced the statistically significant differences (lower $p$ values; Table 4 ) between WT and LDLR-KO rats (Fig. $4 \mathrm{~b}-\mathrm{d}$ ). Overall, these data showed that normalization of aortic contractility to length or volume further improved detection of statistically significant differences and, importantly, had no detrimental effect on statistical outcomes.

\section{Method Validation}

The "proof of principle" tests (i.e., platforms; NC vs. HFD; WT vs. LDLR-KO) support the general applica- 


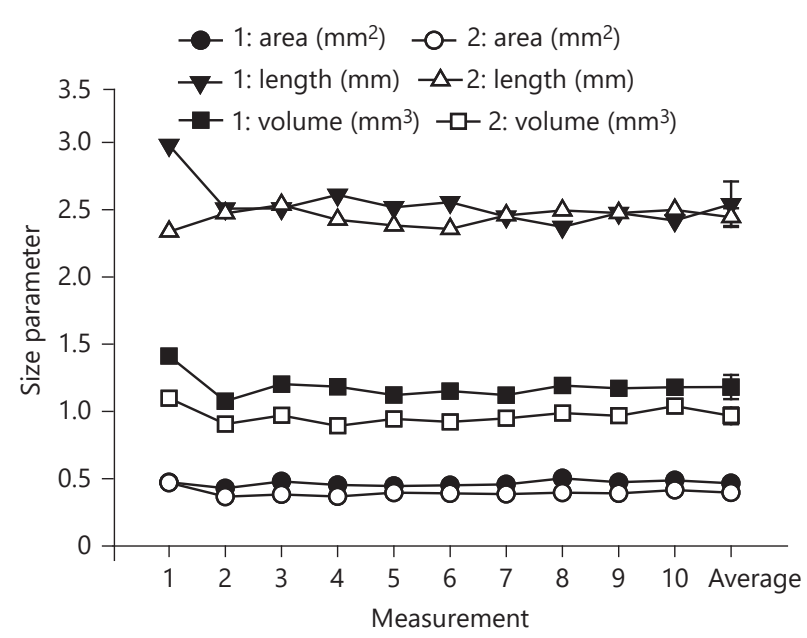

Fig. 5. Reproducibility of aorta parameter data acquisition: intraassay variation. The same images of a blood vessel lengthwise and in cross-section (ring) were repeatedly examined for length and cross-sectional area (x-sec area), respectively, as described above using Image J a total of 10 times by two different observers. Blood vessel volume $\left(\mathrm{mm}^{3}\right)$ was calculated by multiplying length $(\mathrm{mm})$ $\times \mathrm{x}$-sec area $\left(\mathrm{mm}^{2}\right)$. Values $=$ mean \pm SD.

bility of our normalization approach, so we also performed four validation tests. These were: (1) two different "users" quantified the same ring images using ImageJ; (2) two different users quantified the same image multiple times (e.g., 10 times); (3) two different users imaged and then quantified multiple sections from a single aorta; and (4) normalization was performed using segment volume calculated by 2 different methods: (a) our image analysis approach and (b) volume estimated from segment weight (in $\mathrm{mg}$ ) and density ( $\mathrm{mg} /$ $\left.\mu \mathrm{L} ; \mathrm{mg} / \mathrm{mm}^{3}\right)$.

\section{Reproducibility}

Image analysis for length and $\mathrm{x}$-sec area proved highly reproducible (Fig. 5). It appeared that users vary in their first attempt to quantify length, but users rapidly adjusted and subsequently made consistent measurements of the same image. These data support the simple and cost-effective nature of our approach. Images were made with an inexpensive microscope camera (USD < 50) and were analyzed with free imaging software (NIH ImageJ). The approach was independent of the user because measurements made by 2 users of both area
Table 4. Normalization of PE-induced $(10 \mu \mathrm{M})$ isometric contractions in thoracic aortas isolated from wild-type (WT) and LDLRnull (LDLR-KO) 1-year-old Sprague-Dawley rats

\begin{tabular}{lllll}
\hline Group & $\mathrm{mN}$ & $\mathrm{mN} / \mathrm{mm}$ & $\mathrm{mN} / \mathrm{mm}^{2}$ & $\mathrm{mN} / \mathrm{mm}^{3}$ \\
\hline WT & $15.10 \pm 2.55$ & $4.85 \pm 0.66$ & $12.09 \pm 1.59$ & $3.98 \pm 0.52$ \\
LDLR-KO & $22.29 \pm 2.34$ & $8.36 \pm 1.09$ & $19.57 \pm 2.35$ & $7.50 \pm 1.27$ \\
\hline$p$ value & 0.0680 & 0.0283 & 0.0327 & 0.0413 \\
\hline
\end{tabular}

Values $=$ means \pm SE. $n=5,6$ rats, respectively. $p$ values shown are results of unpaired $t$ tests between WT and LDLR-KO groups.

(Fig. 6a) and length (Fig. 6b) using the same 5 blood vessel segments were similar. Although noticeable divergence in volumes between users was present (Fig. 6c), both variability and directionality of values were preserved - indicating both robustness and consistency. Our approach also compared favorably to using blood vessel volume estimated as the quotient of blood vessel mass and using a reported aortic density, i.e. $0.86 \mathrm{mg} / \mathrm{L}$ [12] (Fig. 6d). We think that there is inherently more difficulty in accurately measuring the mass of a wet blood vessel than in using our image-based approach. First, it should be recognized that a short, cleaned segment of mouse aorta weighs approximately $0.1-0.2 \mathrm{mg}$, and a high-quality balance capable of such a measurement is costly. Second, much smaller blood vessels, e.g. mesenteric, may be difficult to weigh accurately because of lumen fluid retention that is difficult to remove without rapidly "drying out" the blood vessel segment [13]. Thus, our low-cost approach is more practical and avoids these pitfalls.

We also tested whether a single blood vessel segment would yield different $\mathrm{x}$-sec areas along its length and whether this was dependent on user evaluation. Although variation in $\mathrm{x}$-sec area was apparent, it appeared less dependent on user and more on image quality and segment location (Fig. 7). These data further emphasize that specific user-based analysis of an image is a less important determinant of $\mathrm{x}$-sec area than is the image quality and blood vessel location (e.g., thoracic aorta tapers proximal to distal). Of course, quality control of image analysis needs to be established in each laboratory adopting this approach. We purposefully used a low cost, highly portable "hobby" or classroom-style digital camera/ microscope to emphasize that even rudimentary optics are sufficient to perform blood vessel analysis, yet practice is required. 


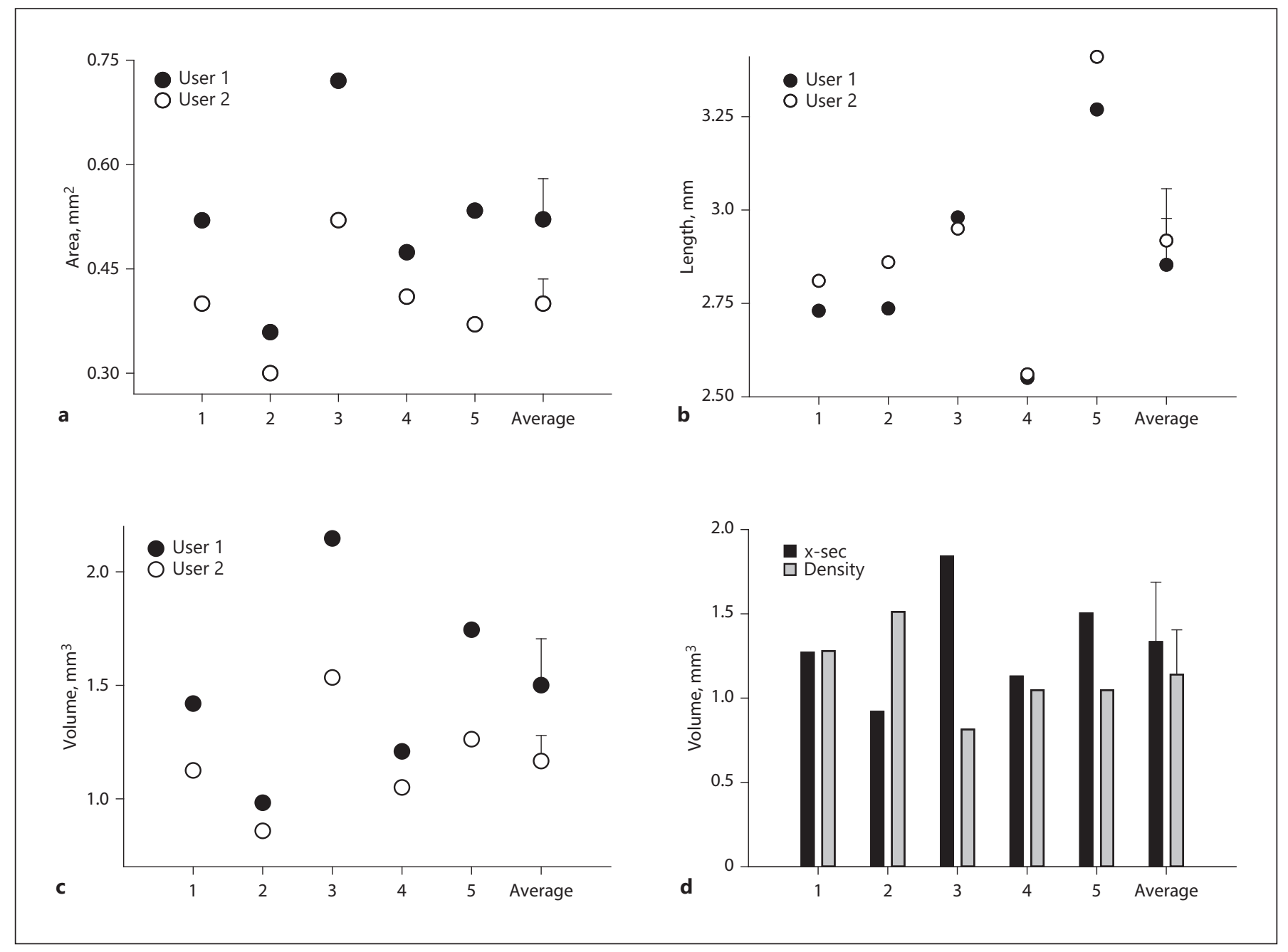

Fig. 6. Reproducibility of aorta parameter data acquisition: inter-assay variation. a Aorta cross-sectional area $\left(\mathrm{mm}^{2}\right)$ measured in five separate aortas independently by two users (user 1; user 2). b Aorta segment length ( $\mathrm{mm}$ ) measured independently by two different users in five separate aortas. c Volume $\left(\mathrm{mm}^{3}\right)$ calculated five times by two users. d Aortic volume calculated from image analysis compared with volume derived from segment weight and aorta density $(0.86 \mathrm{mg} / \mathrm{L})$. Values $=$ mean $\pm \mathrm{SD}$.

\section{Resiliency to Prolonged Formalin Fixation}

Formalin fixation for 1 week (Fig. 8a), 1 month (Fig. 8b), and up to 2 months (Fig. 8c) had little effect on quantified aortic segment volume, so it is feasible to formalin fix a blood vessel and still obtain reliable and robust measurements for use in normalization long after contractility experiments were performed. Additionally, fixed blood vessel segments can be processed, embedded, sectioned, and stained for more refined morphometry and histological staining of the blood vessel wall [14].

\section{Major Advantages and Limitations}

The current study describes a novel method for normalization of vascular contractility in vitro, and highlights 4 major advantages: (1) the method is simple and low cost, and thus, accessible worldwide; (2) the method is validated and is reproducible across 2 different species, platforms and users; (3) the method is resilient to chronic storage of blood vessels in formalin; and (4) if adopted, normalized vascular contraction data with a "universal unit" (either $\mathrm{mN} / \mathrm{mm}$ or $\mathrm{mN} / \mathrm{mm}^{3}$ ) will allow for more across study and across laboratory comparisons, and thus, more efficient use of research resources. 


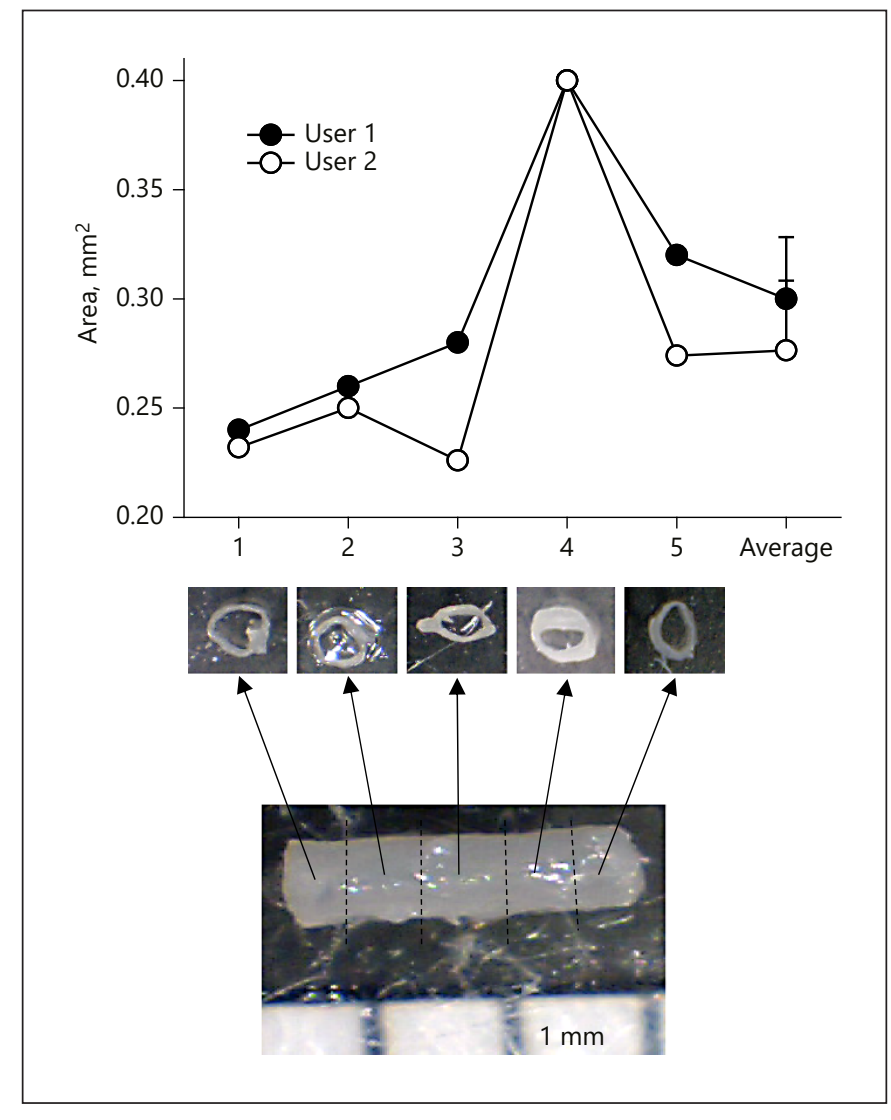

Fig. 7. Intra-aortic variation in the cross-sectional area. A single thoracic aorta segment was cut into five cross-sections ("donut") and each cross-section was imaged and the area measured by two independent users (user 1; user 2). Values $=$ mean \pm SD.

There are several caveats to this method. This method did not use an optimal stretch (i.e., we used $\approx 1 \mathrm{~g}[9.81$ $\mathrm{mN}]$ isometric tension for baseline stretch $[3-5,15]$; optimal tension depends on stretch [16]) or identify the mechanisms of change in contractility (e.g., endothelium dysfunction would still need to be tested). Moreover, it is expected that laboratory conditions likely will vary in many ways that meaningfully contribute to real differences in contractility (e.g., buffer composition, loading tension, temperature, inhibitors, denuding, etc...), but "all things being equal," this approach provides a common denominator, which currently is not used by the vast majority of laboratories. As indicated above, the method reduces variability in raw values by correcting for differences that arise as a function of blood vessel segment size. Blood vessel segments may differ in size simply due to randomness or because two different laboratories use different platforms. An obvious limitation is that we only

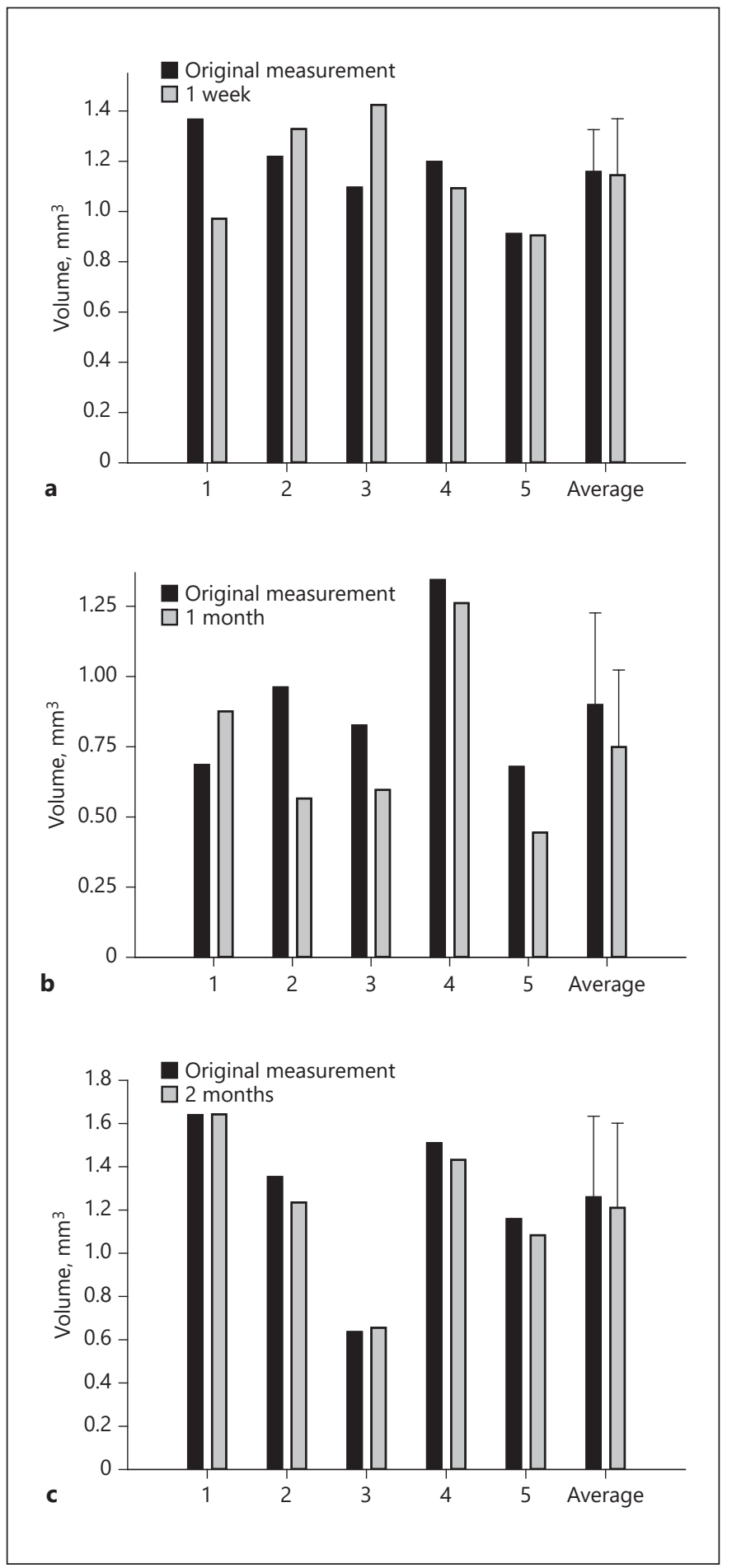

Fig. 8. Resiliency of aorta parameter data acquisition over time. Comparison of aorta volumes quantified after formalin fixation of the blood vessel. a Aorta volume measured 1 week after formalin fixation. b Aorta volume measured 1 month after formalin fixation. c Aorta volume measured 2 months after formalin fixation. Values $=$ mean \pm SD. 
tested the protocol using aortas (albeit both from mice and rats); however, the method will likely work in other blood vessels because there is an inherent reliance of contraction on vascular smooth muscle that makes up the bulk of the media in aorta and all other arteries as well [14]. In any case, we predict that normalization (with segment length and/or volume) is superior to using only raw contraction values. In one of our real-world examples, we detected significant changes in aortic contractility with HFD feeding (vs. NC) only when we normalized contractions (Fig. 3). This outcome has value because importantly it reduces the overall cost of a study by reducing the number of animals needed in a study. Finally, our approach was found to be robust, consistent, reproducible, and resilient. It is recommended that isometric contractions of isolated aorta be normalized to either blood vessel segment length $(\mathrm{mN} / \mathrm{mm})$ and/or volume $\left(\mathrm{mN} / \mathrm{mm}^{3}\right)$ to both improve quantitative assessment of contractility and foster across study comparisons. In future studies, this normalization method can be applied in other blood vessels as and when validated for each vascular bed.

\section{Acknowledgements}

The technical assistance of M. Bertke, A. Kelleher, W. McKinley, D.W. Riggs, and G. Shirk is acknowledged. We thank Dr. S. Srivastava for use of rat aorta data. This study was supported by NIH grants GM103492, HL122676, and ES014559.

\section{Disclosure Statement}

No potential conflicts of interest relevant to this article were reported.

\section{References}

1 Zhang Z, Wang M, Fan XH, Chen JH, Guan YY, Tang YB: Upregulation of TRPM7 channels by angiotensin II triggers phenotypic switching of vascular smooth muscle cells of ascending aorta. Circ Res 2012;111:11371146.

2 Li P, Zhu N, Yi B, Wang N, Chen M, You X, Zhao X, Solomides CC, Qin Y, Sun J: MicroRNA-663 regulates human vascular smooth muscle cell phenotypic switch and vascular neointimal formation. Circ Res 2013;113: 1117-1127.

3 Conklin DJ, Haberzettl P, Prough RA, Bhatnagar A: Glutathione-S-transferase P protects against endothelial dysfunction induced by exposure to tobacco smoke. Am J Physiol Heart Circ Physiol 2009;296:H1586-H1597.

4 Conklin DJ, Boor PJ: Allylamine cardiovascular toxicity: evidence for aberrant vasoreactivity in rats. Toxicol Appl Pharmacol 1998;148: 245-251.

5 Conklin DJ, Boyce CL, Trent MB, Boor PJ: Amine metabolism: a novel path to coronary artery vasospasm. Toxicol Appl Pharmacol 2001;175:149-159.

6 Reynolds S, Williams AS, Williams H, Smale S, Stephenson HJ, Amos N, George SJ, O'Donnell VB, Lang D: Contractile, but not endothelial, dysfunction in early inflammatory arthritis: a possible role for matrix metalloproteinase-9. Br J Pharmacol 2012;167: 505-514.
7 Schepelmann M, Yarova PL, Lopez-Fernandez I, Davies TS, Brennan SC, Edwards PJ, Aggarwal A, Graca J, Rietdorf K, Matchkov V, Fenton RA, Chang W, Krssak M, Stewart A, Broadley KJ, Ward DT, Price SA, Edwards DH, Kemp PJ, Riccardi D: The vascular $\mathrm{Ca}^{2+}$ sensing receptor regulates blood vessel tone and blood pressure. Am J Physiol Cell Physiol 2016;310:C193-204.

8 Kim SK, Avila JJ, Massett MP: Strain survey and genetic analysis of vasoreactivity in mouse aorta. Physiol Genomics 2016;48:861873.

9 Conklin DJ, Cowley HR, Wiechmann RJ, Johnson GH, Trent MB, Boor PJ: Vasoactive effects of methylamine in isolated human blood vessels: role of semicarbazide-sensitive amine oxidase, formaldehyde, and hydrogen peroxide. Am J Physiol Heart Circ Physiol 2004;286:H667-H676.

10 Sithu SD, Malovichko MV, Riggs KA, Wickramasinghe NS, Winner MG, Agarwal A, Hamed-Berair RE, Kalani A, Riggs DW, Bhatnagar A, Srivastava S: Atherogenesis and metabolic dysregulation in LDL receptor-knockout rats. JCI Insight 2017;2:86442.

11 Silva JF, Correa IC, Diniz TF, Lima PM, Santos RL, Cortes SF, Coimbra CC, Lemos VS: Obesity, inflammation, and exercise training: relative contribution of iNOS and eNOS in the modulation of vascular function in the mouse aorta. Front Physiol 2016;7:386.
12 Weind KL, Ellis CG, Boughner DR: Aortic valve cusp vessel density: relationship with tissue thickness. J Thorac Cardiovasc Surg 2002;123:333-340.

13 Murphy RA: Mechanics of vascular smooth muscle. Compr Physiol DOI: 10.1002/cphy. cp020213.

14 Conklin DJ, Kong M, HEI Health Review Committee: Part 4. Assessment of plasma markers and cardiovascular responses in rats after chronic exposure to new-technology diesel exhaust in the ACES bioassay. Res Rep Health Eff Inst 2015:111-139; discussion 141-171.

15 Wheat LA, Haberzettl P, Hellmann J, Baba SP, Bertke M, Lee J, McCracken J, O'Toole TE, Bhatnagar A, Conklin DJ: Acrolein inhalation prevents vascular endothelial growth factorinduced mobilization of Flk-1+/Sca-1+ cells in mice. Arterioscler Thromb Vasc Biol 2011; 31:1598-1606.

16 Wendling WW, Krasner LJ, Cooper SC, Chen D, Harakal C, Addonizio VP, Brister NW, Carlsson C: Effects of stretch or distention on phenylephrine-induced constriction of human coronary artery bypass grafts. J Cardiothorac Vasc Anesth 2001;15:717-722. 